\title{
Practical Considerations of Using the K3 Camera in CDS Mode for High-resolution and High-throughput Single Particle Cryo-EM
}

Ming Sun, Caleigh Azumaya, Eric Tse, Daniel Southworth, Kliment Verba, Adam Frost, Yifan Cheng and David Agard

University of California, San Francisco, San Francisco, California, United States

A recent technological breakthrough in electron cryo-microscopy (cryo-EM) is the development of direct electron detection cameras for data acquisition. Earlier iterations, such as the GATAN K2 camera, have provided single electron detection at practical dose rates and resulted in dramatic improvements in detective quantum efficiency (DQE) and reduction in noise. Most recently, the next generation of direct detector has become widely available, with 1.6x increased detector size, 3.75x faster internal frame rate, and higher DQE. Moreover, the GATAN K3 camera also successfully implemented correlative double sampling (CDS) mode during standard data collection.

In this study we set out two objectives, to determine if data collected on the K3 detector are affected by dose rate and/or the use of the newly implemented correlative double sampling mode (CDS) and to determine if cryo-EM density maps can be reconstructed to highest resolution using an image shift data collection scheme to increase throughput. In CDS mode, the effective camera frame rate is reduced by half compared with non-CDS mode. Our data show that a lower dose rate ( 8 electrons per pixel per second (eps) vs 16eps) increases the quality of reconstructions for both apoferritin and aldolase test samples. This is likely because of increasing coincidence loss with increasing dose rate. When coincidence loss is held constant between the two imaging modes, i.e. 8 eps CDS and 16 eps non-CDS modes, our result indicates that data collected in CDS-mode reconstructs to higher resolution. When comparing CDS and non-CDS modes under the same imaging condition, the gain in resolution using CDS is partially offset by higher coincidence loss and the gain in resolution is not as robust, but is still present. We further analyzed the influence of pixel size and compared a large, highly symmetric sample and a smaller, less symmetric sample. Our results will be interesting to the cryo-EM community for deciding on an optimal data collection strategy for different projects.

References

J Struct Biol. 2013 Nov; 184(2): 10.1016

Nat Methods. 2013 Jun; 10(6): 584-90.

Microscopy (Oxf). 2016 Feb; 65(1): 35-41 